\title{
Critical Analysis of Electronic Simulation of Financial Market Fluctuations
}

\author{
H. Fanchiotti, C.A. García Canal and N. Martínez * \\ Departamento de Física, Universidad Nacional de La Plata \\ C.C. 67 - 1900 La Plata, Argentina.
}

November 20, 2018

\begin{abstract}
An interesting analog circuit for simulating a signal with fluctuations having a probability density function with a power tail has recently been proposed and constructed. The exponent of the power law can be fixed by tuning an appropriate circuit element. The proposal is to use the circuit as a simulatorgenerator of financial market fluctuations and as a tool for risk estimations and forecasts. We present a discussion of the stability conditions for multiplicative noise and an exhaustive analysis of the power law fluctuation generator in connection with the electronic components and their parameters. From our studies one can conclude that the proposal is not adequate to provide a confident experimental scheme to follow the fluctuations of the financial market due to both electronic implementation and to difficulties in parameter tuning.
\end{abstract}

*E-mails: huner@fisica.unlp.edu.ar; garcia@fisica.unlp.edu.ar; nolo@fisica.unlp.edu.ar 


\section{Introduction}

Recently, Sato, Takayasu and Sawada [1] have constructed a very interesting analog circuit able to generate a signal corresponding to a time series with fluctuations resembling those of a typical financial market. The corresponding probability density function presents a power law tail.

It is worth mentioning that the proposal of Ref. [1] immediately attracted the opinion of the scientific community. In particular, we should mention that the American Institute of Physics Bulletin of Physics News number 478 of April 6, 2000 includes a comment on Ref. [1] under the title "An electrical circuit mimics yen-dollar fluctuations". It ends by saying that the circuit costing approximately $\$ 5$ can estimate yen-dollar fluctuations as fast as the $\$ 10,000$ workstations that are running mathematical simulations of the exchange rates. On the other hand, CERN Courier of June 2000 includes also a comment under the title "How can you make a million?" where one can read that Japanese Scientists have unveiled a $\$ 5$ electrical circuit that can mimic fluctuations in the yen-dollar exchange rate.

The circuit constructed in Ref. [1] being very interesting in its own, deserves a careful analysis from the electronic point of view. We present here an exhaustive study of the circuit behavior and of its potentialities for reproducing financial market fluctuations. We conclude that the circuit really mimics these fluctuations but the meaning of this mimics is precisely the one the Oxford Dictionary gives: imitate closely in order to make fun of.

Before the presentation of the circuit and its relationship with the Langevin equation with multiplicative noise, we discuss, in Section 2 the stability conditions for this kind of noise. Afterwards we include in Section 3 an analysis of the power law fluctuations generator in connections with the electronic components and their corresponding parameters. A circuit simulation completes our study and its results are presented in Section 4. Finally, Section 5 summarizes our conclusions.

\section{Stability}

Before entering into the discussion of both the electronic and the simulation analysis, let us briefly review the stability in multiplicative stochastic processes. It is well known that an additive stochastic process is stable whenever the associated deterministic problem is. In fact, given the process defined by

$$
\frac{d v}{d t}=L(v)+\eta
$$


where $\eta$ is a standard Gaussian noise, it has a stable stationary behaviour when all its moments $\left\langle v^{n}\right\rangle$ exist up to the n-th order. This imposes a condition on the integral of $L(v)$ [2].

However, for a multiplicative noise the stability of the deterministic problem is not enough to ensure the stability under fluctuations [2]. Consider now the process

$$
\frac{d v}{d t}=-d v+v \eta
$$

where $\eta$ is again a standard Gaussian noise and $d$ a parameter. In this case the moments are given by

$$
\left\langle v^{n}\right\rangle=\left\langle v^{n}\right\rangle_{t=0} \exp [-n t(d-n D / 2)]
$$

where $D$ is the measure of the Gaussian fluctuation, namely

$$
\langle\eta(t+\tau) \eta(t)\rangle=D \delta(\tau)
$$

The stationary distribution results

$$
p_{0}(v)=v^{-1-2 d / D}
$$

which is not normalizable. It is then clear from Eq. (3) that in this case of multiplicative noise, the condition

$$
n \leq \frac{2 d}{D}
$$

has to be fulfilled in order to guarantee the finiteness of the moments $\left\langle v^{n}\right\rangle$ when $t \rightarrow \infty$. Consequently, if $d<D / 2$, the multiplicative noise overcome the deterministic restoring term $-d v$, even if this deterministic problem has a stable solution.

Having these mathematical conditions in mind, we re-analyzed the circuit proposed in Ref [1]. The main point is to simulate, electronically or numerically, the equation of Langevin type with multiplicative noise (2), that in the present case reads

$$
\frac{d v_{0}}{d t}=\left(-\frac{1}{R_{f} C_{f}}+\frac{k}{R_{v} C_{f}} \mu(t)\right) v_{0}+\psi(t)
$$

Notice a change of sign in the first term of the r.h.s of this equation, with respect to Eq. (12) of Ref. [1]. 


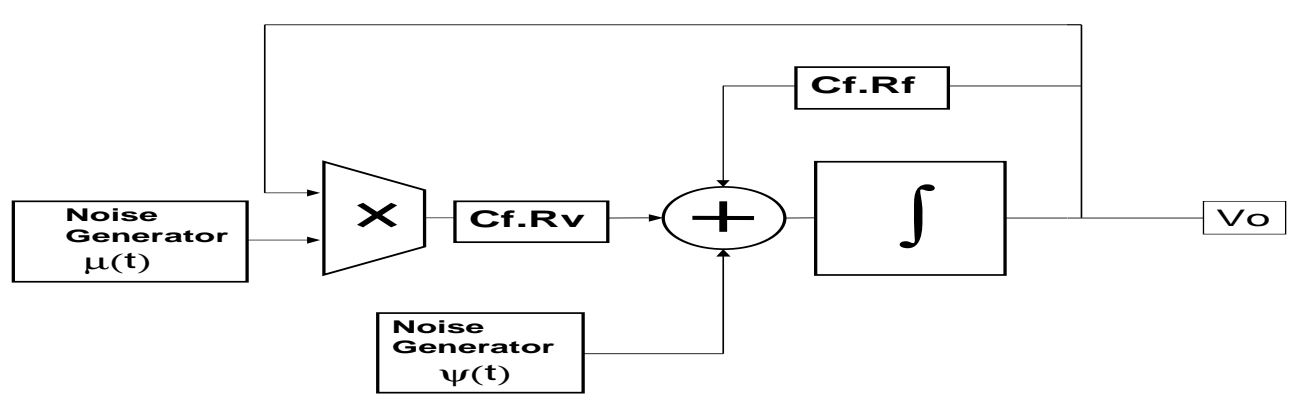

Figure 1: Block diagram of analog simulation

\section{Electronics}

\subsection{Generalities}

The general block diagram in Fig. 11 represents the integrated form of the original differential equation 5 .

The different blocks involved in Fig. 1 are implemented by using electronic components, mainly amplifiers. From the diagram in Fig. 1, the electronic implementation can be easily derived giving rise to the circuit in Fig. 2

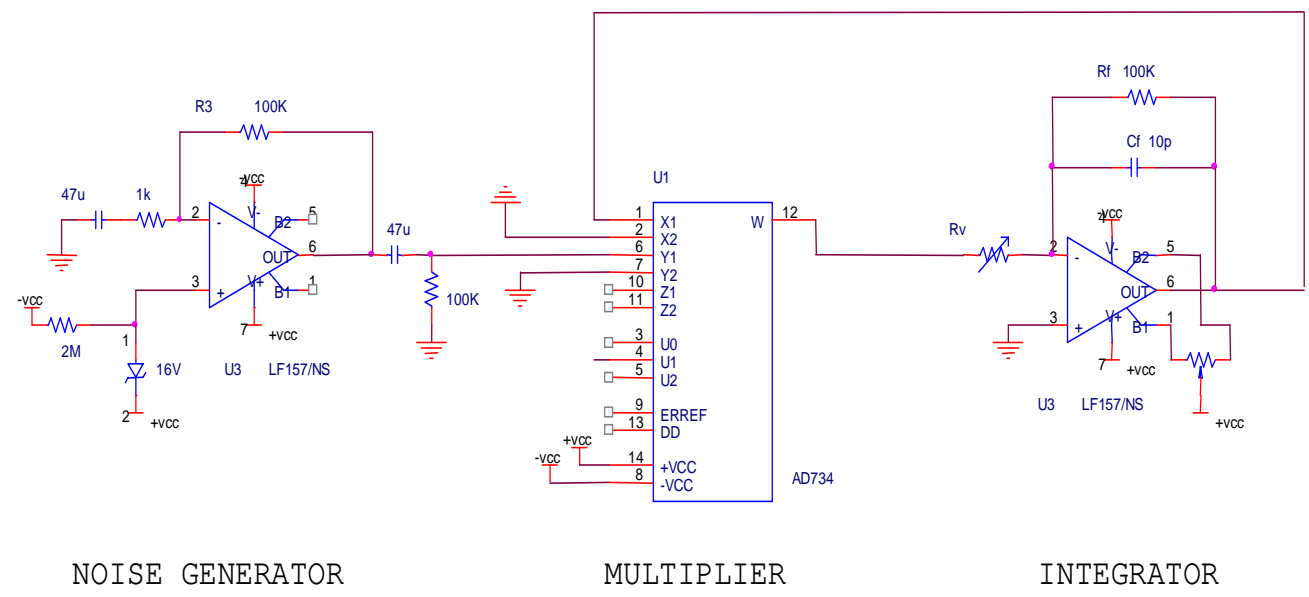

Figure 2: Power fluctuation generator

The electronic components have their own characteristics and impose 
some restrictions on the functions to be implemented. It means that the range of values where the function is valid is bounded by the electronic components behaviour.

Let us then analyze, following Ref. [3], the integrator block implementation shown in Fig. 3 .
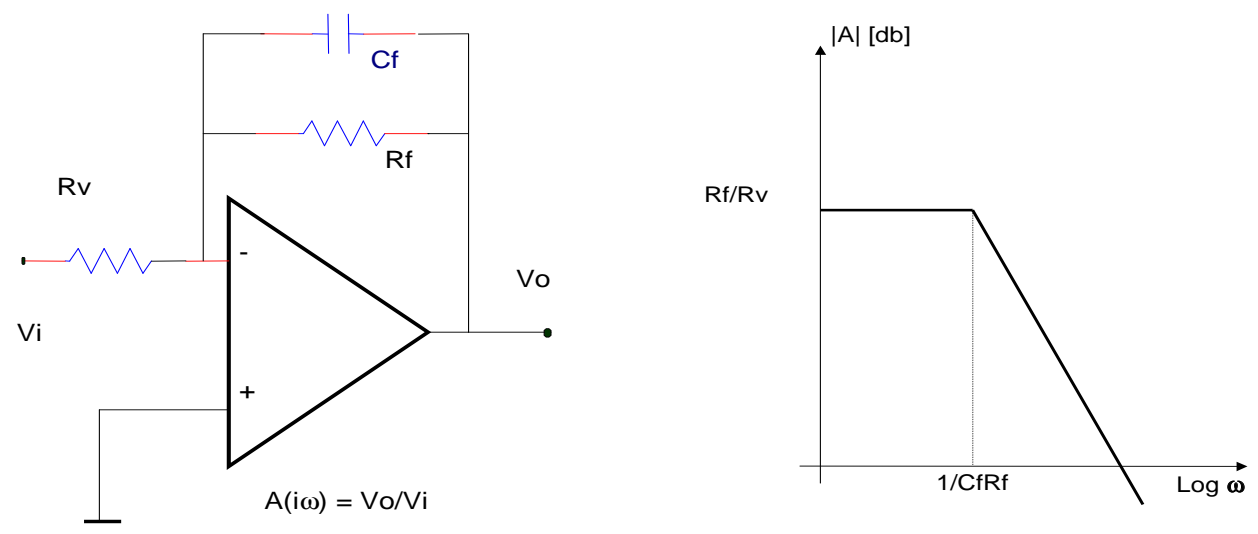

Figure 3: Electronic integrator and its Bode plot

Considering an ideal amplifier, namely one with Gain Product Bandwith $(\mathrm{GBW}) \rightarrow \infty$ and Gain $\left(A_{0}\right) \rightarrow \infty$, the corresponding transfer function is

$$
A(s)=\frac{V_{0}(s)}{V_{i}(s)}=-\frac{R_{f}}{R_{v}} \frac{1}{\left(1+s R_{f} C_{f}\right)}
$$

with $s=i \omega$. Fig. 3 includes also the Bode plot of this expression.

Under the condition $\omega \gg 1 /\left(C_{f} R_{f}\right)$, Eq (6) gives rise to

$$
V_{0}(s)=-\frac{R_{f}}{R_{v}} \frac{1}{\left(s R_{f} C_{f}\right)} V_{i}(s)
$$

and

$$
v_{0}(t)=-\frac{1}{R_{f} C_{f}} \int v_{i}(t) d t
$$

Consequently the above circuit works as an integrator for frequencies above $\omega_{p}=1 /\left(R_{f} C_{f}\right)$. In summary, to implement an integrator using a real operational amplifier (OA) its GBW must be larger than the highest frequency of interest and $A_{0} \gg R_{f} / R_{v}$.

To verify the above conditions, the Bode plot of the desired integrator, should be compared with that of the open loop gain of the OA. As a rule of thumb, the implementation will be valid if the plot of the OA "contains" the integrator's Bode plot. 


\subsection{Circuit analysis}

The criteria above was applied to the original circuit proposed in Ref. [1] that includes the values

$$
C_{f}=10 p F ; \quad R_{f}=100 K \Omega ; R_{v}=5-200 \Omega
$$

giving rise to

$$
\omega_{p}=1 M H z ; A=\frac{R_{f}}{R_{v}}=54-86 d b .
$$

In Fig. 因, a Bode diagram of Eq. (6) is plotted together with the OA LF 157 characteristic obtained from the National Semiconductor Co. Linear Databook. As can easily be seen, the condition that the OA "contains" the desired characteristic is not fulfilled. So the changes of $R_{v}$ between the specified values do not produce a full change in the "integrator gain" and the observed effects are only marginal ones. In other words, a change in $R_{v}$ is not fully reflected as a change of the "integrator gain".

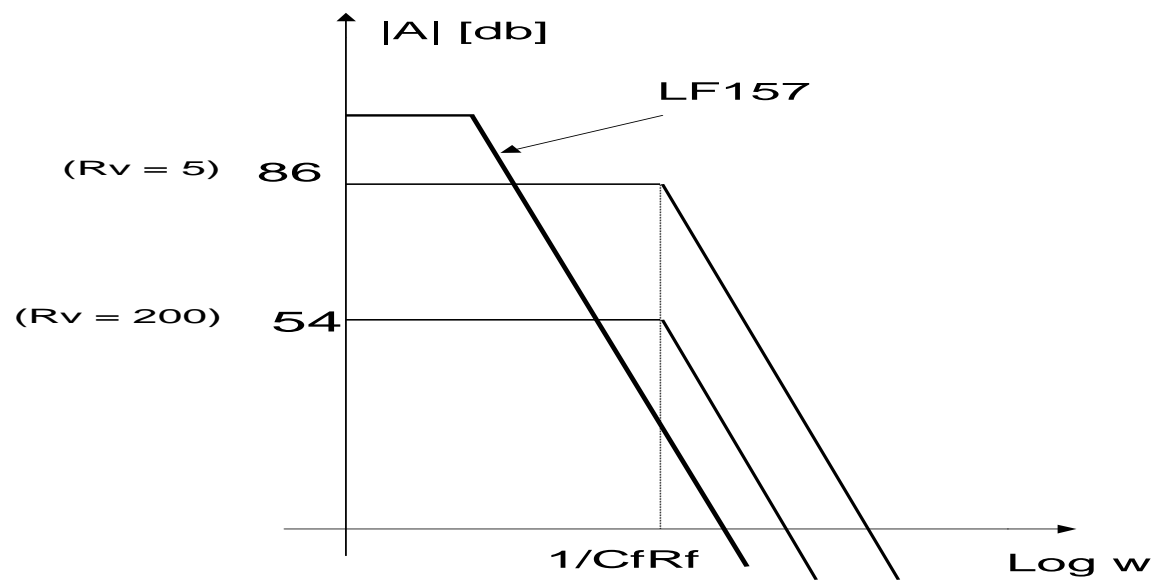

Figure 4: Comparison between Bode plots for the amplifier and the integrator

Moreover, when the circuit in Fig. 2 was tested with the specified values of $R_{v}$, the output presents unwanted saturation as shown in Fig. 5 .

Only for values of $R_{v}>1.7 \mathrm{~K} \Omega$, the amplifier remains almost linear giving rise to the output seen in Fig. 6. In fact, the reported behaviour in 


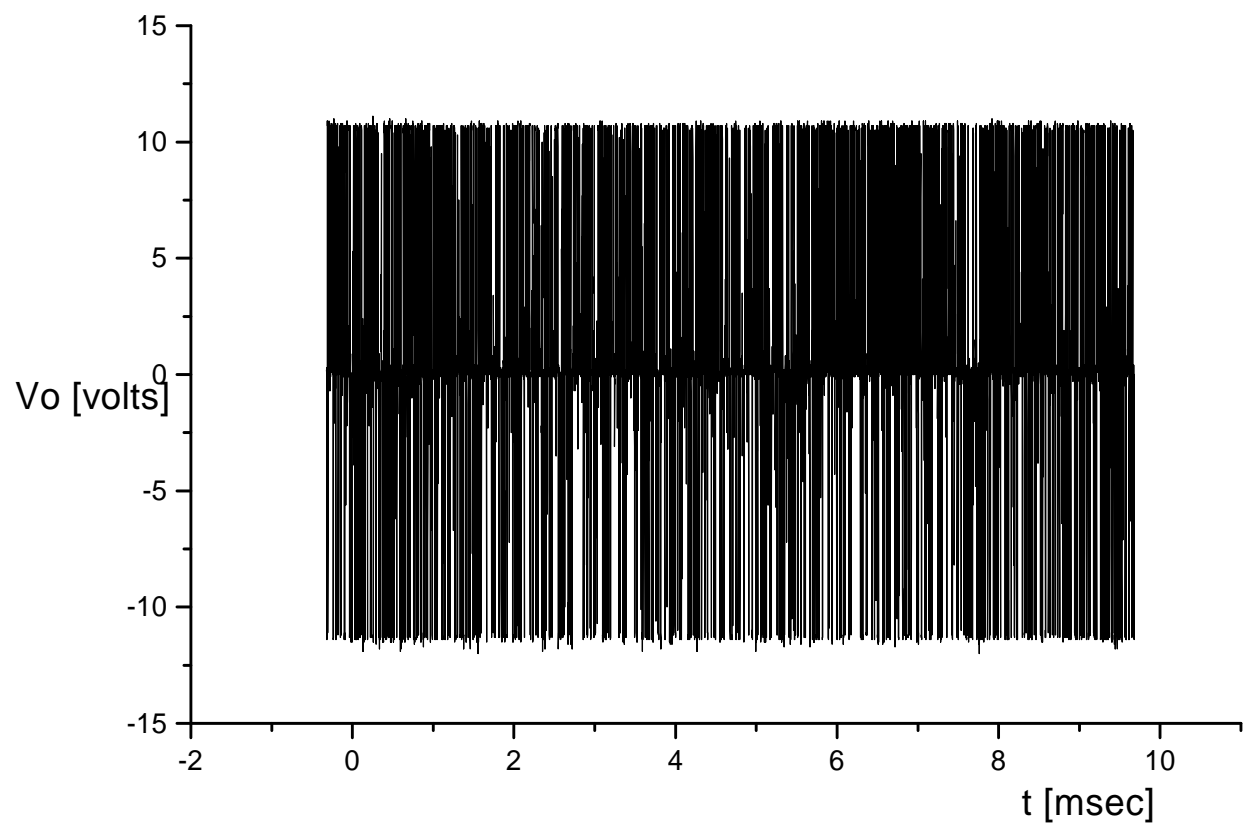

Figure 5: Output of the circuit with the original parameters for $R_{v}=35 \Omega$

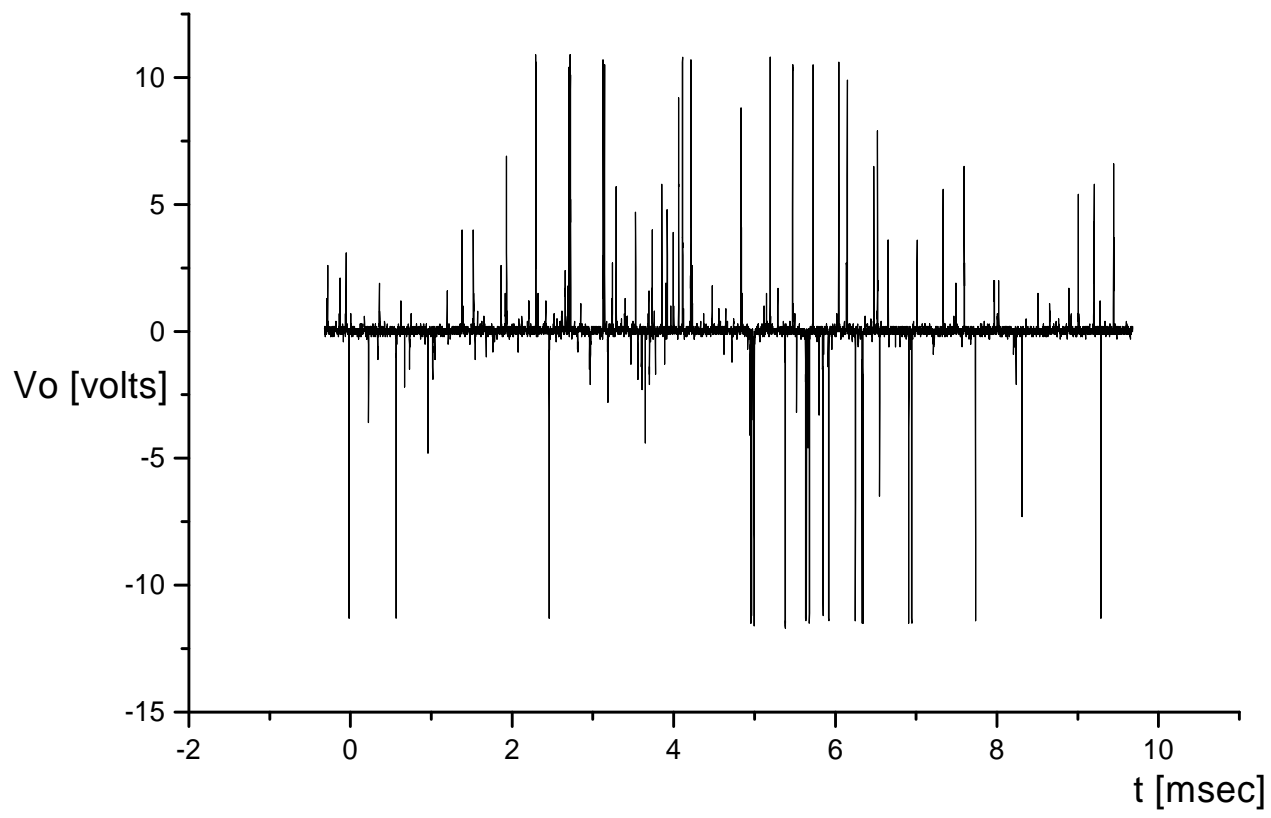

Figure 6: Output of the circuit with the original parameters for $R_{v}=2200 \Omega$ 
Ref. [1] was qualitative found for $1.7 K \Omega<R_{v}<4 K \Omega$. For larger values, the electrical noise dominates.

It is important to remark that our measurements were done with a Tek scope (Model TDS 3030) with 8 bits of resolution. Nevertheless we are certain that this technical detail doesn't change our conclusions.

Following the circuit criteria discussed above, a new set of values for $R_{v}$, $C_{f}$ and $R_{f}$ was chosen . To include the integrator characteristic inside the Operational Amplifier (OA) open loop, a lower pole frequency $\left(\omega_{p}\right)$ was in order. To avoid output saturation, higher values of $R_{v}$ are also needed. Then, we decided to use the values

$$
C_{f}=11 p F ; R_{f}=800 K \Omega ; R_{v}=6-12 K \Omega \text {. }
$$

Resulting in

$$
\omega_{p}=113 \mathrm{KHz} ; A=\frac{R_{f}}{R_{v}}=35-45 \mathrm{db}
$$

This is not an arbitrary selection because a value of $C_{f}$ higher than $15 \mathrm{pF}$ put the system in a heavy oscillation. A careful study of the LF157 characteristics shows that the stability conditions are greatly affected if a capacitor larger than $15 p F$ is connected between the input and the output. Consequently, a different amplifier should be used instead.

The circuit with modified parameters worked as predicted. In Fig. 7 we present the log-log plot of the probability density function for the $R_{v}$ values specified.

Using the adequate values for $C_{f} R_{f}$, a tighter control of the integrator gain is obtained. Notice also that for lower values of $R_{v}$, that means higher gain, the circuit does not met the integrator condition, and for $R_{v}$ higher than $12 K \Omega$ the electrical noise dominates.

\section{Simulation}

In order to check the influence of the amplifier's offset voltage we have performed additional tests. This offset, independently of the circuit values, does not affect the final power law behaviour and can be reduced in the data processing stage.

If the circuit is to be really useful in predicting fluctuations on a quantitative way, it must be necessary to know the influence of each electrical parameter on the output signal in order to reproduce, for example, the probability distribution of historical data. This work would be tedious, when not impossible using the real circuit. 


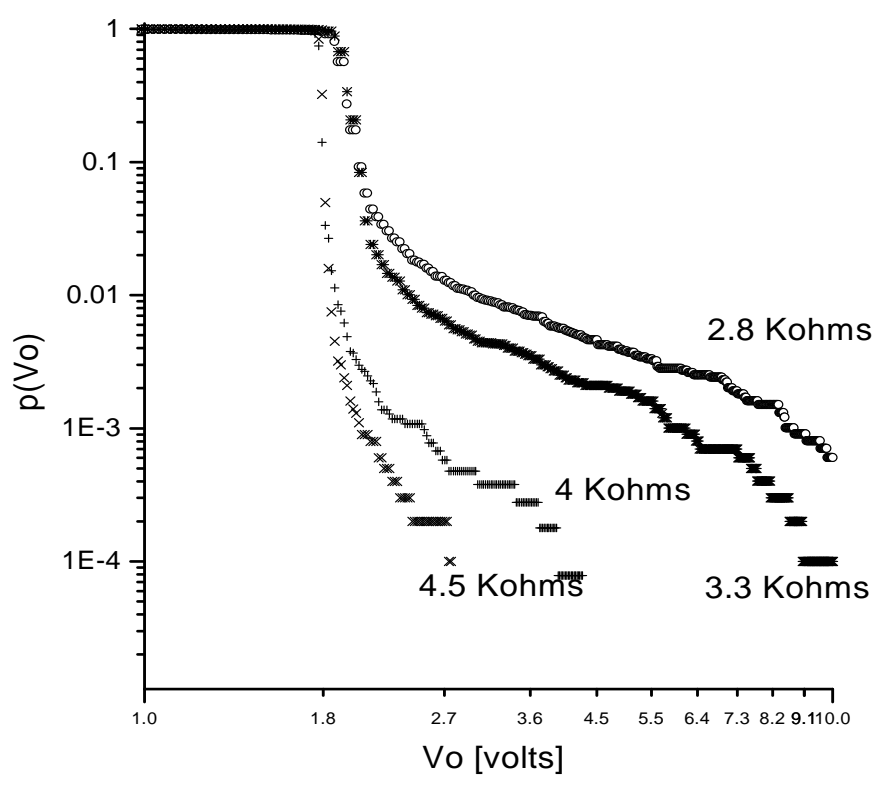

Figure 7: Probability density function for the modified parameters

We propose to use instead some simulation procedures that, without loosing the electrical point of view, put in evidence the influence of each of the parameters. We have tried several configurations using the Spice program [四]. Although this program allows one to use realistic components, by means of transistor- level models, we prefer to use quite ideal representation where the different parameters of the circuit, desirable or not, can be added separately. It can be mentioned that the simulation with real components simulation is also plagued of serious convergence problems, being certainly difficult to make it runs in a fiable way.

Fig. 8 shows the circuit used in the simulation. It reproduces the differential equation of interest. Different gain blocks allows one to change amplitudes, parameters such as offset voltage and saturation limits when added, as well as different noise generators.

The typical output of the simulation is summarized in Fig. 9.

\section{Conclusions}

We have carefully analyzed the recently proposed electronic analog circuit that generates fluctuations with a probability density function having power law tails. 


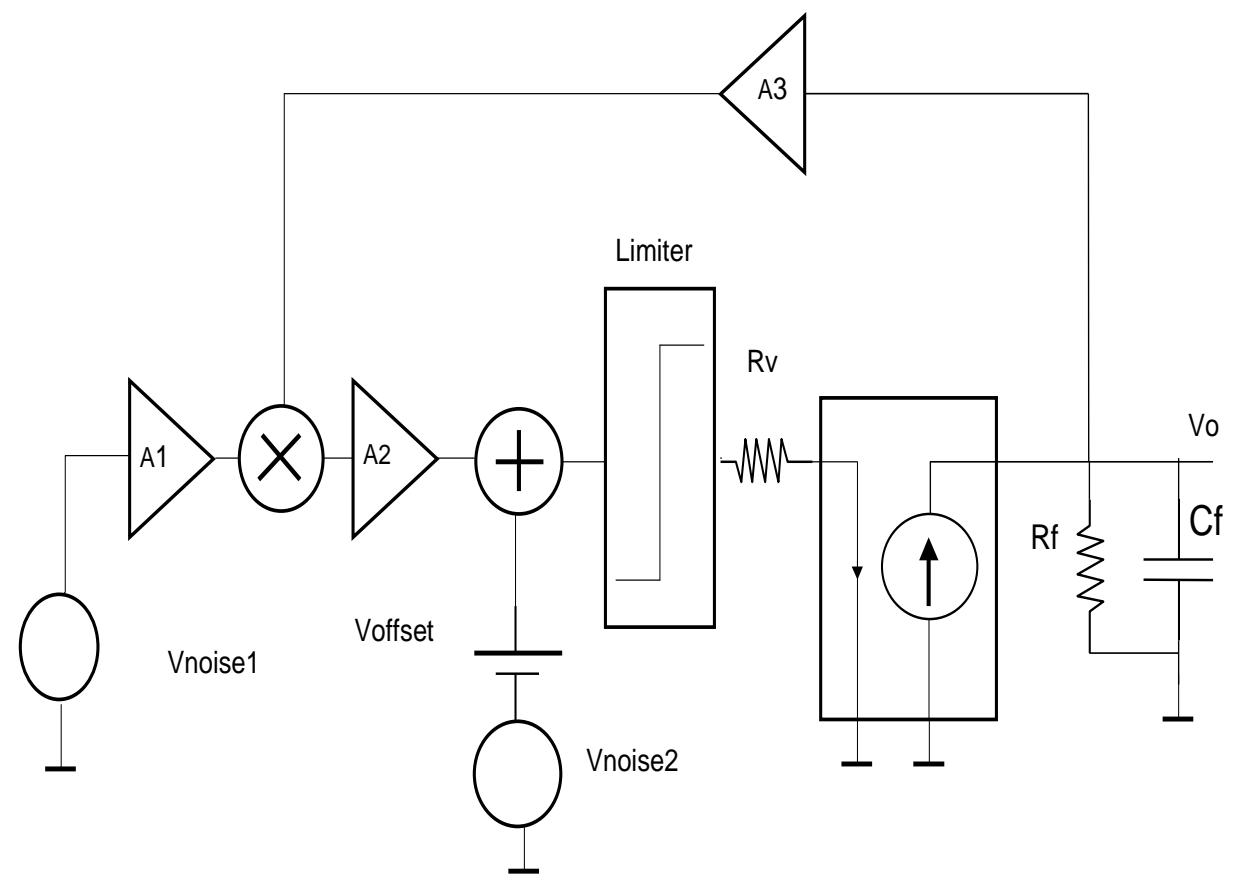

Figure 8: Simulated circuit

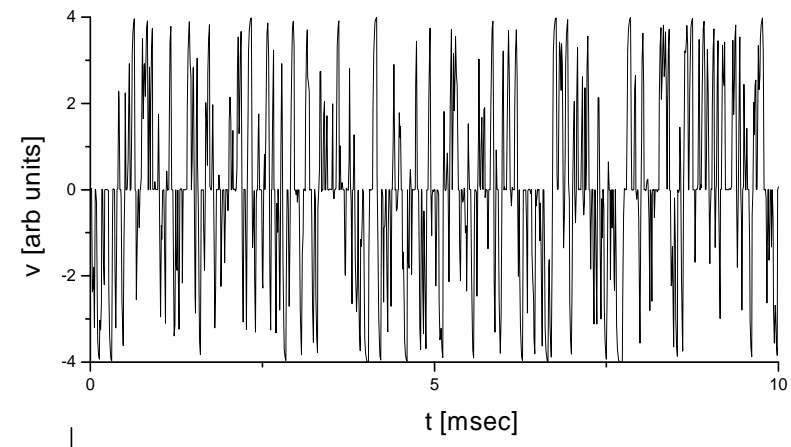

(a)

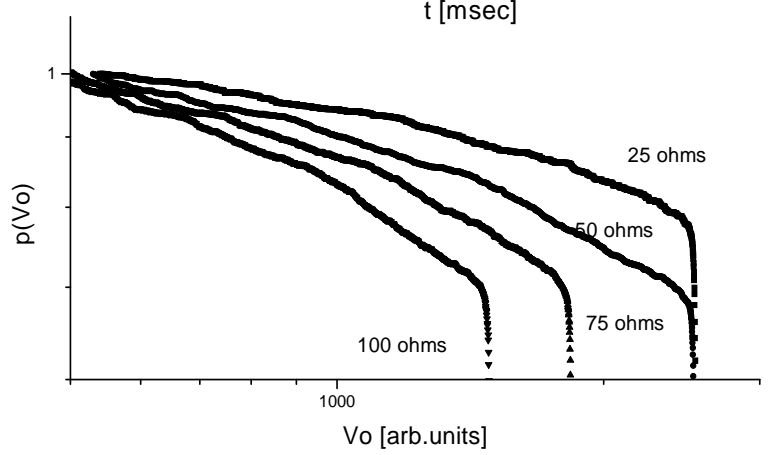

(b)

Figure 9: Output of the simulation. a)signal; b) density of probability 
We found that the originally proposed parameters are not the most pertinent ones because they place the circuit in the borderline of the integrator Bode plot. With appropriate parameters, the circuit performance is noteworthily improved. Moreover, by changing the integrated circuit originally chosen, a further improvement can be achieved.

On the other hand, the numerical simulation, even if carried out in terms of ideal electronic components, reinforces the previous conclusions.

Finally, several comments are in order:

- Even if the power fluctuation generator circuit is certainly a low cost apparatus, a costly peripheral equipment is needed for taking and analyzing the output data.

- The circuit, in order to mimic reasonable fluctuations, needs a fine tuning of the pertinent parameters.

- A direct numerical simulation of the original equation seems to be a much better solution.

\section{Acknowledgment}

We want to warmly thank A.-H. Sato for correspondence regarding reference [1].

\section{References}

[1] A. Sato, H. Takayasu, Y. Sawada, Fractals 8219 (2000).

[2] A. Schenzle, H. Brand, Physical Review A20, 1628 (1979).

[3] P. Horowitz, W. Hill, The Art of Electronics. Cambridge University Press (1980).

[4] MicroSim PSPICE version 6.2, MicroSin Co. 1995. 